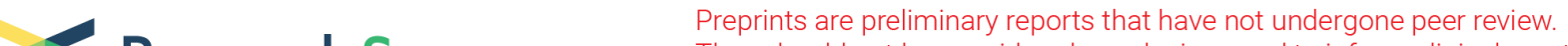 Research Square They should not be considered conclusive, used to inform clinical practice, or referenced by the media as validated information.
}

\section{A Rare Case of Recurrent Ovarian Cancer With NTRK1-TPM3 Gene Rearrangement}

\section{Yuta Endo ( $\nabla$ yenyen@fmu.ac.jp )}

Fukushima Medical University School of Medicine https://orcid.org/0000-0002-4227-0530

\section{Takafumi Watanabe}

Fukushima Medical University School of Medicine

\section{Motonobu Saito}

Fukushima Medical University School of Medicine

\section{Katsuharu Saito}

Fukushima Medical University School of Medicine

\section{Rei Suzuki}

Fukushima Medical University School of Medicine

\section{Hideki Sano}

Fukushima Medical University School of Medicine

\section{Yutaka Natori}

Fukushima Medical University School of Medicine

\section{Eisaku Sasaki}

Fukushima Medical University School of Medicine

\section{Makiko Ueda}

Fukushima Medical University School of Medicine, Fukushima

\section{Norihito Kamo}

Fukushima Medical University School of Medicine, Fukushima

\section{Shigenori Furukawa}

Fukushima Medical University School of Medicine, Fukushima

\section{Shu Soeda}

Fukushima Medical University School of Medicine, Fukushima

Koji Kono

Fukushima Medical University School of Medicine, Fukushima

\section{Shigehira Saji}

Fukushima Medical University School of Medicine, Fukushima

\section{Keiya Fujimori}

Fukushima Medical University School of Medicine, Fukushima

\section{Research Article}


Keywords: NTRK gene fusion, NTRK1-TPM3, Entrectinib, Next-generation sequencing, Immunohistochemistry, Ovarian cancer

Posted Date: September 16th, 2021

DOI: https://doi.org/10.21203/rs.3.rs-739649/v2

License: (c) (1) This work is licensed under a Creative Commons Attribution 4.0 International License. Read Full License 


\section{Abstract}

Background: NTRK gene fusion is rare in gynecologic cancer. Entrectinib is a novel targeted drug which is a potent inhibitor of TRK A, B and C. Here, we present a case of recurrent ovarian cancer with NTRK1TPM3 rearrangement, which was detected by next-generation sequencing (NGS) and treated with entrectinib.

Case Presentation: A 56-year-old woman was diagnosed as having stage IV ovarian cancer due to pleural effusion. Neoadjuvant chemotherapy and interval debulking surgery followed by chemotherapy were performed. Ten months after completion of chemotherapy, the patient's disease recurred. She was treated with multimodal therapy for recurrence. DNA-based NGS detected NTRK1-TPM3 rearrangement and entrectinib was started. However, the patient's disease progressed despite six weeks' administration of entrectinib, and one month after discontinuation of entrectinib, she died. After her death immunohistochemistry with a pan-Trk monoclonal antibody was performed to determine the expression of TRK. However, immunohistochemistry was negative for TRK.

Conclusion: We presented a rare case of recurrent ovarian cancer with NTRK1-TPM3 gene fusion, in which entrectinib was not effective. While NTRK gene fusion was detected by DNA-based NGS, immunohistochemistry was negative for TRK. Immunohistchemitory should be performed for confirmation of TRK protein expression before entrectinib administration.

\section{Background}

NTRK gene fusions are consistently detected in rare types of cancers (secretory breast carcinoma, mammary analogue secretary carcinoma, congenital infantile fibrosarcoma, and congenital mesoblastic nephroma) and they are novel therapeutic targets across multiple tumor types. [1-3] On the other hand, these gene fusions are rare in common adult cancers. $[1,2]$ In gynecologic oncology, NTRK gene fusion is also rare, although there are several reports of uterine sarcoma with this fusion gene. [4-7] A previous cohort study showed that NTRK1-TPM3 is most frequent in NTRK1 fusions across multiple histologies. [2] Immunohistochemistry (IHC) staining, fluorescence in situ hybridization, reverse transcriptase polymerase chain reaction, DNA-based next-generation sequencing (NGS) and RNA-based NGS are used to identify patients with NTRK gene fusion cancer. Each method to detect NTRK gene fusion has its own characteristics. [3]

Entrectinib is a potent inhibitor of TRKA, TRKB, TRKC, ROS1, and ALK, and is specifically designed to have systemic activity. In gynecologic oncology, treatment using entrectinib is rare because of the low frequency of NTRK fusions. [1] Here, we report a case of recurrent ovarian cancer (OC) with NTRK1-TPM3 gene fusion and that was treated with entrectinib.

\section{Case Presentation}


In September 2013, a 56-year-old woman was referred to our hospital with bilateral ovarian tumor, multiple disseminations in the peritoneum, bilateral pleural effusion, and multiple swellings of the pelvic, and paraaortic lymph nodes. Her serum level of cancer antigen 125 (CA125) was elevated to $1740 \mathrm{U} / \mathrm{ml}$. She was diagnosed as having stage $\triangle \mathrm{OC}$ according to the International Federation of Gynecology and Obstetrics (FIGO) 1988 because pleural effusion cytology was positive. Paclitaxel $\left(175 \mathrm{mg} / \mathrm{m}^{2}\right)$ and carboplatin (area under the curve 6) were started as neoadjuvant chemotherapy. After four courses of chemotherapy, computed tomography (CT) revealed a reduction in tumor size. Interval debulking surgery including abdominal hysterectomy, bilateral salpingo-oophorectomy, omentectomy, and pelvic and paraaortic lymphadenectomy, was performed. Histopathological diagnosis was high-grade serous carcinoma. Following this surgery, another three courses of the same regimen were administered, and the patient achieved clinical complete response.

Ten months after the last therapy, OC recurred. Because of the disease persistence, she was treated with nine regimens of chemotherapy combined with two surgeries for recurrent tumors; the first was partial hepatectomy due to dissemination to the liver, and the second was enterectomy due to recurrence in the mesentery. Microsatellite stability was detected in specimens from the second recurrence resection.

Six years after initiation of therapy, FoundationOne ${ }^{\circledR} \operatorname{CDx}$ (Foundation Medicine, Cambridge, MA), which is DNA based NGS and covers 324 genes, was performed based on the patient's archival tumor tissue from the second recurrence resection. This revealed a missense variant of TP53 (c.731G $>A$ ) and a rearrangement between exon 11 of NTRK1 (NM_002529) and somewhere around exon 2-3 of TPM3 (pos1="chr1:156844554-156844771", pos2="chr1:154155588-154155822"). Oral entrectinib $(600 \mathrm{mg} /$ day) was started after discussing with experts. Six weeks after initiation of entrectinib, the patient's serum CA125 level elevated to $4360 \mathrm{U} / \mathrm{ml}$, although it was $1712 \mathrm{U} / \mathrm{ml}$ before initiation of entrectinib and CT revealed progression of liver metastasis. (Fig. 1) Adverse events during entrectinib administration comprised grade 2 dysgeusia. One month after discontinuation of entrectinib, the patient died. (Fig. 2)

After the patient's death, IHC staining with a pan-Trk monoclonal antibody (mAB) clone EPR17341 (Abcam, Cambridge, MA) was performed to assess for TRKA, TRKB, and TRKC expression as previously described. [8] This $m A B$ clone is most commonly used and has been investigated thoroughly. In addition, this $\mathrm{mAB}$ clone reacts with a conserved proprietary peptide from the $\mathrm{C}$-terminus of TRKA, TRKB and TRKC, and is therefore reactive to any oncogenic NTRK fusion. [3] IHC was negative for all specimens from the primary site resection, as well as the first and second recurrent site resections. (Fig. 3)

\section{Discussion}

Here we presented a case of recurrent OC with NTRK1-TPM3 rearrangement. Additionally, the present case demonstrates the discrepancy between gene rearrangement detected by NGS and protein expression. This discrepancy may be a biomarker for predicting the ineffectiveness of entrectinib for cancers with NTRK rearrangement detected by NGS. 
In the current case, NGS revealed NTRK1-TPM3 rearrangement and a missense variant of TP53. There are few approved therapies for TP53 variants, although almost all cases of ovarian high-grade serous carcinoma (95\%) have somatic TP53 variants. [9] On the other hand, NTRK fusions are oncogenic drivers and novel targets. Doebele et al. reported the safety and activity of entrectinib in adult patients with advanced or metastatic NTRK fusion-positive cancer across three clinical trials (ALKA-372-001, STARTRK1 and STARTRK-2). In these trials, only one ovarian cancer patient was included. They showed that the objective response rate, which included complete response and partial response, was $57 \%(95 \% \mathrm{Cl} 43.2-$ 70.8). The median duration of response was 10 months ( $95 \% \mathrm{Cl} 7.1$ to not estimable) and the percentage of progressive disease (PD) was only $7 \%$. However, the characteristics of cases with PD remained unclear in their report. [1]

In the present case, entrectinib was administered because NGS revealed NTRK1-TPM3 rearrangement and entrectinib was recommended after a discussion among experts. However, this novel target drug was ineffective. TRK protein was not expressed, although IHC testing with a pan-Trk mAB clone [EPR17341] was performed. A previous study reported that gene fusions involving NTRK1, 2, and 3 and their partner genes result in a constitutive activation or overexpression of TRK receptors, potentially leading to oncogenesis. [10] Additionally, other reports have shown that pan-Trk IHC yielded a sensitivity of 75$95.2 \%$, and a specificity of $92-100 \%$ and that the sensitivity of pan-Trk IHC for TRKA was $96.2 \%$. [3, 8, 11, 12] Pan-Trk IHC is a reliable screening method for the detection of NTRK gene fusions based on this date. Moreover, pan-Trk IHC can rapidly assess malignancies which may harbor possible NTRK gene fusions in order to determine eligibility of patients for targeted therapy with TRK inhibitors. [8] However, it should be considered that there are NTRK rearrangements which are found to be negative by $\mathrm{IHC}$, and can only be detected by NGS, such as in the present case.

Drilon et al. reported the efficacy of larotrectinib, which is a selective inhibitor of TRKA, TRKB and TRKC. In their study, six of an initial 55 patients showed primary resistance to larotrectinib. Three patients had tumor material available for central analysis, and in all three cases, pan-Trk IHC did not reveal the presence of TRK protein expression. This indicated that the rearrangements detected by NGS were false positives or that the identified fusion genes were not expressed at the protein level. [13] It is considered that entrectinib has the same characteristics as larotrectinib with regard to discrepancy between gene fusion and protein expression, as observed in the current case, and that this finding may be a key to predict the ineffectiveness of entrectinib for cancers with NTRK rearrangement detected by NGS.

To the best our knowledge, this is the first case report of OC with NTRK rearrangement. It is known that a small percentage of common adult cancers carry fusions of NTRK genes. [2] A large cohort study revealed that the frequency of NTRK gene fusions was $0.25 \%$ of general cancers. $[2,12]$ Therefore, physicians have few chances to experience this molecular characteristic. However, physicians should be aware of the pitfall that TRK protein may not expression even if NGS reveals NTRK rearrangement.

\section{Conclusion}


In conclusion, we here presented a rare case of recurrent OC with NTRK1-TPM3 fusion. Physicians should be aware of the discrepancy of DNA rearrangement and protein expression, and IHC should be performed for confirmation of TRK protein expression before entrectinib administration.

\section{Abbreviations}

IHC: immunohistochemistry

NGS: next-generation sequencing

OC: ovarian cancer

CA-125: cancer antigen 125

FIGO: International Federation of Gynecology and Obstetrics

CT: computed tomography

mAB: monoclonal antibody

PD: progressive disease

\section{Declarations}

Ethics approval and consent to participate.

This study was approved by the Ethical Committee of Fukushima Medical University.

\section{Consent for publication}

Written informed consent was obtained from the patient for publication of this case report and the accompanying images. A copy of the written consent is available for review by the Editor-in-Chief of this journal.

\section{Availability of data and materials}

The data used or analyzed are all included in this published article

\section{Competing interests}

The authors declare that they have no competing interests.

\section{Funding}

We have no commercial or financial incentives associated with the publication of this article. 


\section{Author's contribution}

YE, TW and MS drafted the manuscript. YE, TW, RS, HS, YN, ES, MU, NK, SF, SS, SS and KF managed this patient. MS, KS and KK performed immunohistochemical staining. All authors have read and approved the final manuscript.

\section{Acknowledgement}

Not applicable.

\section{References}

1. Doebele RC, Drilon A, Paz-Ares L, et al. Entrectinib in patients with advanced or metastatic NTRK fusion-positive solid tumours: integrated analysis of three phase 1-2 trials. Lancet Oncol. 2020;21(2):271-82.

2. Getalica Z, Xiu J, Swensen J, et al. Molecular characterization of cancers with NTRK gene fusions. Mod Pathol. 2019;32:147-53.

3. Solomon JP, Benayed R, Hechtman JF, et al. Identifying patients with NTRK fusion cancer. Ann Oncol. 2019;30(Suppl 8):viii16-22.

4. Chiang S, Cotzia P, Hyman DM, et al. NTRK Fusions Define a Novel Uterine Sarcoma Subtype With Features of Fibrosarcoma. Am J Surg Pathol. 2018;42(6):791-8.

5. Rabban JT, Devine WP, Sangoi AR, et al. NTRK fusion cervical sarcoma: a report of three cases, emphasising morphological and immunohistochemical distinction from other uterine sarcomas, including adenosarcoma. Histopathology. 2020;77(1):100-11.

6. Hodgson A, Pun C, Djordjevic B, et al. NTRK-rearranged Cervical Sarcoma: Expanding the Clinicopathologic Spectrum. Int J Gynecol Pathol. 2021;40(1):73-7.

7. Croce S, Hostein I. McCluggage WG. NTRK and other recently described kinase fusion positive uterine sarcomas: A review of a group of rare neoplasms. Genes Chromosomes Cancer. 2021;60(3):147-59.

8. Hechtman JF, Benayed R, Hyman DM, et al. Pan-Trk Immunohistochemistry Is an Efficient and Reliable Screen for the Detection of NTRK Fusions. Am J Surg Pathol. 2017;41(11):1547-51.

9. Boyarskikh UA, Gulyaeva LF, Avdalyan AM, et al. Spectrum of TP53 Mutations in BRCA1/2 Associated High-Grade Serous Ovarian Cancer. Front Oncol. 2020;16:10:1103.

10. Khotskaya YB, Holla VR, Farago AF, et al. Targeting TRK family proteins in cancer. Pharmacol Ther. 2017;173:58-66.

11. Rudzinski ER, Lockwood CM, Stohr BA, et al. Pan-Trk Immunohistochemistry Identifies NTRK Rearrangements in Pediatric Mesenchymal Tumors. Am J Surg Pathol. 2018;42(7):927-35.

12. Solomon JP, Linkov I, Rosado A, et al. NTRK fusion detection across multiple assays and 33,997 cases: diagnostic implications and pitfalls. Mod Pathol. 2020;33(1):38-46. 
13. Drilon A, Leatsch TW, Kummar S, et al. Efficacy of Larotrectinib in TRK Fusion-Positive Cancers in Adults and Children. N Engl J Med. 2018;378(8):731-9.

14. Munkhdelger J, Shimooka T, Koyama Y, et al. Basaloid Squamous Cell Carcinoma of the Uterine Cervix: Report of a Case With Molecular Analysis. Int J Surg Pathol. 2021. doi:10.1177/1066896921997132.

\section{Figures}
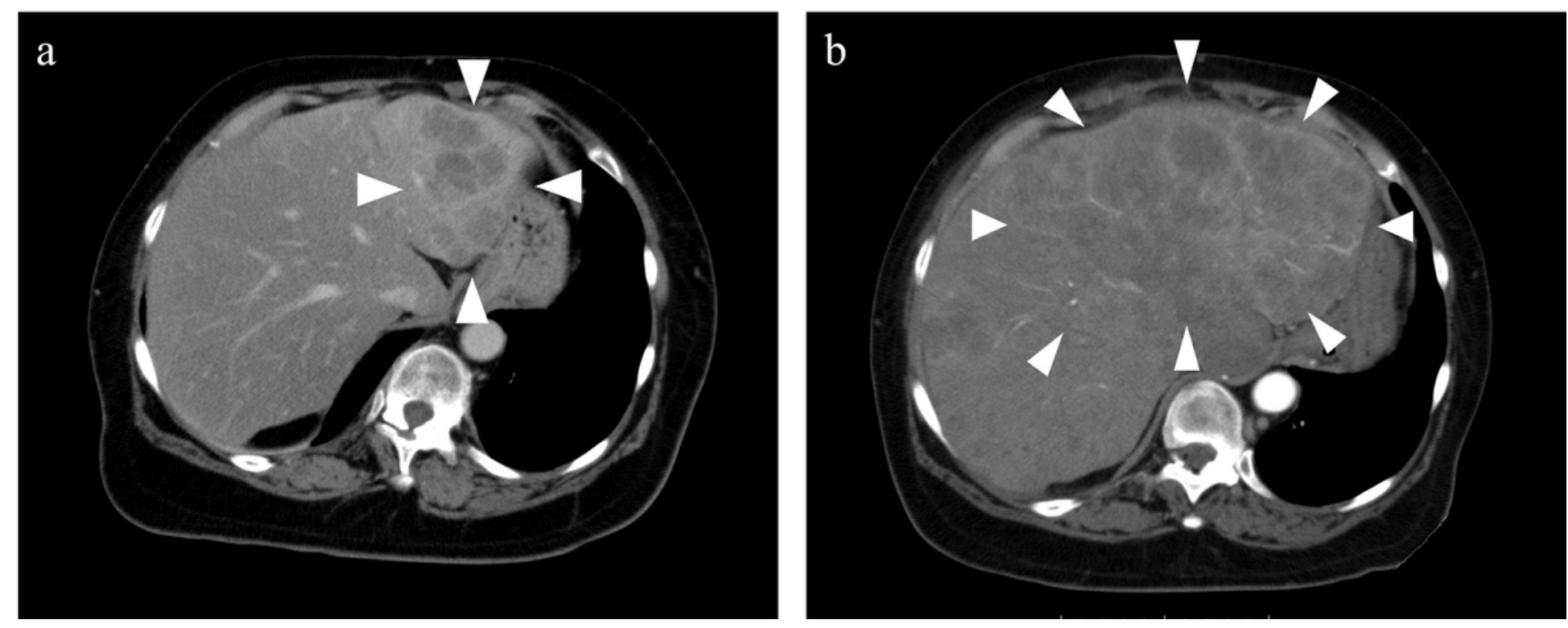

\section{Figure 1}

Computed tomography of liver metastasis a. Before administration of entrectinib b. Six weeks after beginning administration of entrectinib. 


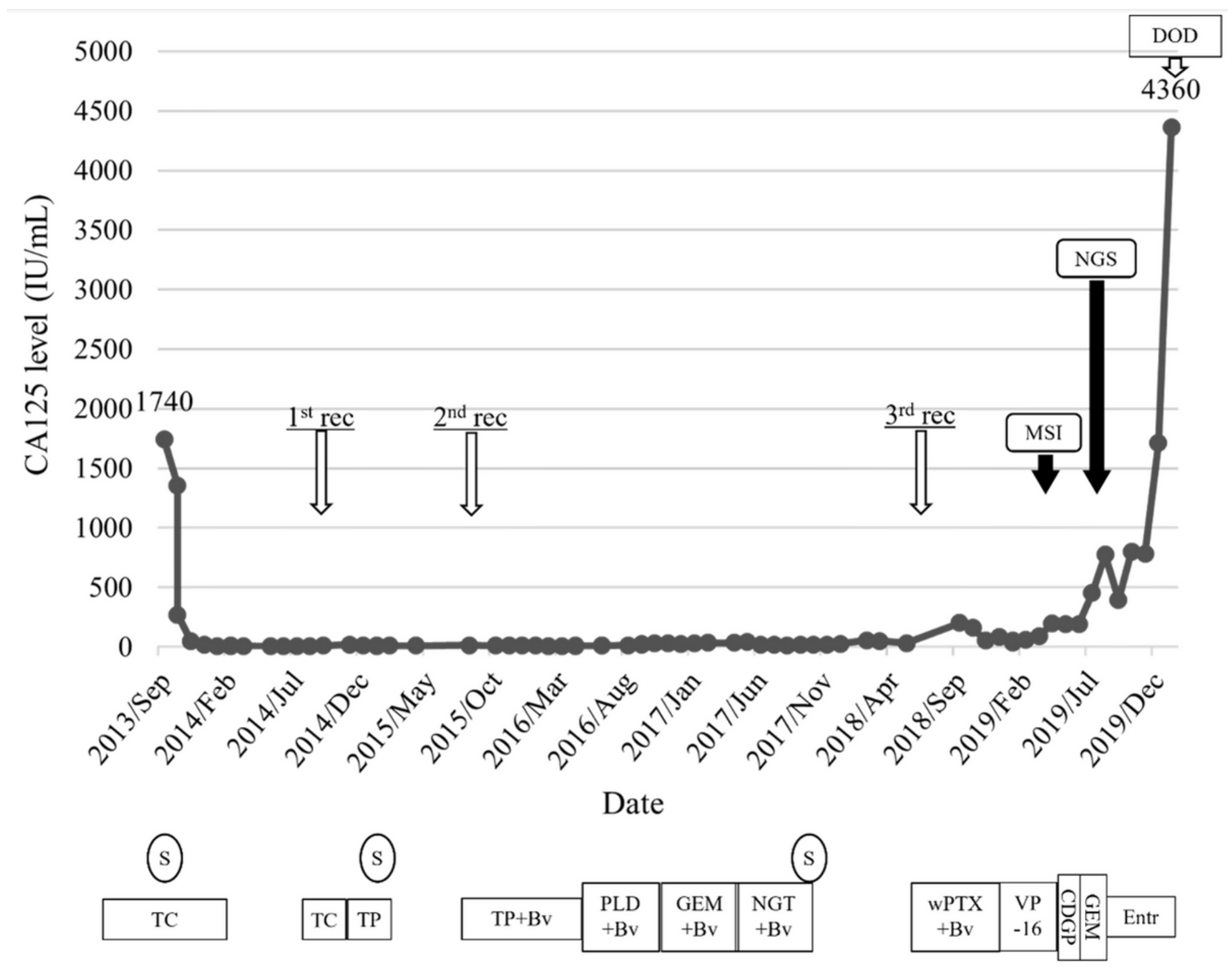

Figure 2

Clinical flowchart of this patient CA125: Cancer antigen-125; MSI: microsatellite instability test; NGS: next generation sequencing; DOD: dead of disease; S, surgery; TC, paclitaxel and carboplatin; TP, paclitaxel and cisplatin; BV, bevacizumab; PLD, pegylated liposomal doxorubicin; GEM, gemcitabine; NGT, nogitecan; wPTX, weekly paclitaxel; VP-16, etopocide; CDGP, nedaplatin; Entr, entrectinib. 

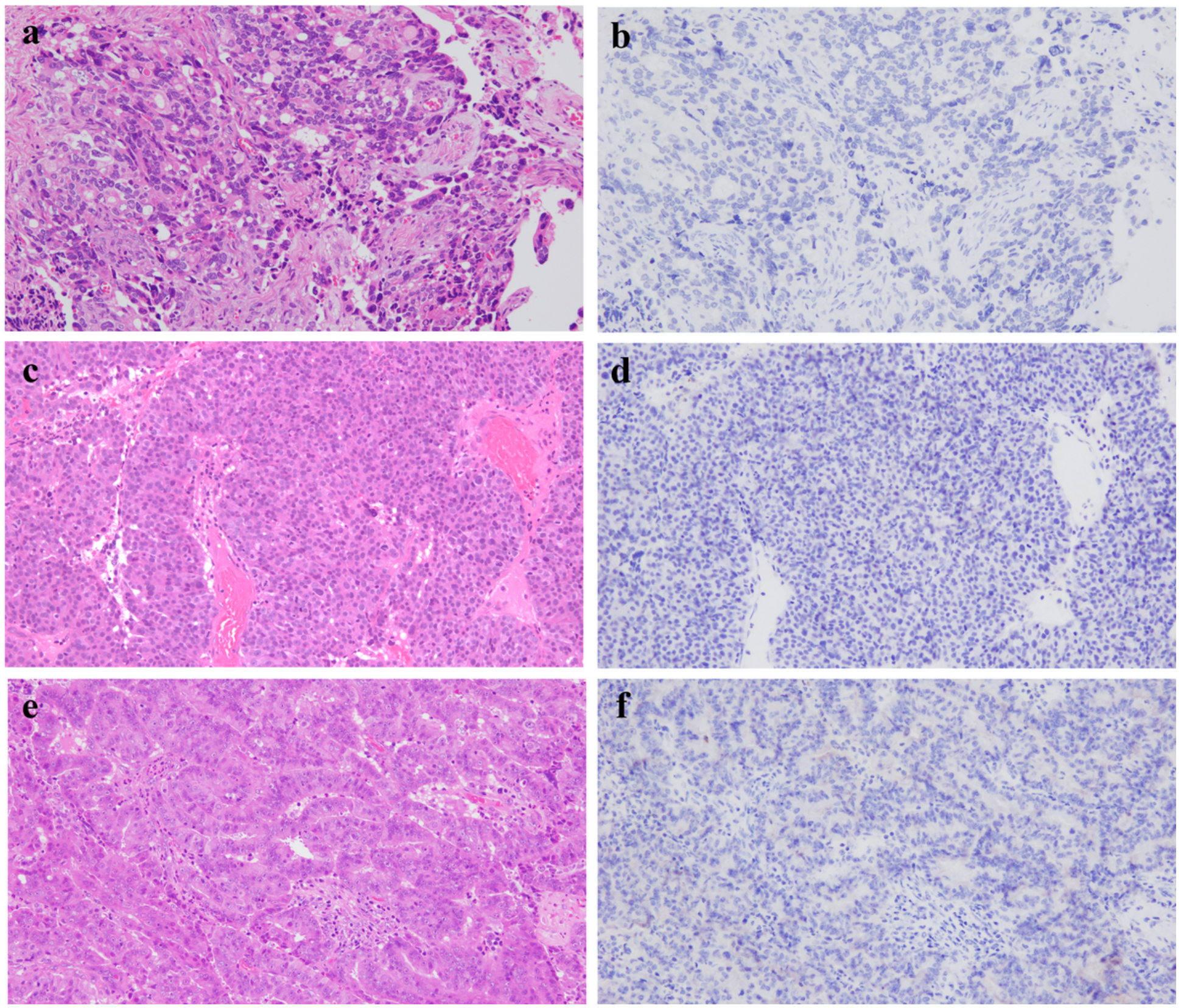

\section{Figure 3}

Histopathological and immunohistochemical features $(\times 100) a, b$. Interval debulking surgery specimen (tumor resection following neoadjuvant chemotherapy) c, d. Liver dissemination specimen (first resection for recurrence) e, f. Mesentery dissemination specimen (second resection for recurrence) a, c, e. Hematoxilin and eosin staining b, d, f. Immunohistochemical stain for pan-Trk. Immunohistochemistry was negative for pan-TRK among all specimens. 